\title{
Local character of magnetic coupling in ionic solids
}

\author{
Ibério de P. R. Moreira and Francesc Illas \\ Departament de Química Física i Centre Especial de Recerca en Química Teòrica, Universitat de Barcelona, C/Martí i Franquès 1, \\ 08028 Barcelona Spain \\ Carmen J. Calzado and Javier F. Sanz \\ Departamento de Química Física, Universidad de Sevilla, C/Prof. García González s/n, 41006 Sevilla, Spain \\ Jean-Paul Malrieu, Nadia Ben Amor, and Daniel Maynau \\ Laboratoire de Physique Quantique, URA 505, Université Paul Sabatier, 118 route de Narbonne, 31062 Toulouse Cedex, France
}

(Received 5 October 1998)

\begin{abstract}
Magnetic interactions in ionic solids are studied using parameter-free methods designed to provide accurate energy differences associated with quantum states defining the Heisenberg constant $J$. For a series of ionic solids including $\mathrm{KNiF}_{3}, \mathrm{~K}_{2} \mathrm{NiF}_{4}, \mathrm{KCuF}_{3}, \mathrm{~K}_{2} \mathrm{CuF}_{4}$, and high- $T_{c}$ parent compound $\mathrm{La}_{2} \mathrm{CuO}_{4}$, the $J$ experimental value is quantitatively reproduced. This result has fundamental implications because $J$ values have been calculated from a finite cluster model whereas experiments refer to infinite solids. The present study permits us to firmly establish that in these wide-gap insulators, $J$ is determined from strongly local electronic interactions involving two magnetic centers only thus providing an $a b$ initio support to commonly used model Hamiltonians. [S0163-1829(99)51510-5]
\end{abstract}

Since its introduction by Heisenberg in 1928 (Ref. 1) and operator formulation by Dirac and Van Vleck in the early thirties, ${ }^{2}$ the spin, or Heisenberg Hamiltonian, has been invariably used to describe isotropic magnetic interactions between localized spin moments. For two particles having total spin $S$ the Heisenberg Hamiltonian has a simple form

$$
\hat{H}^{\text {Heisenberg }}=-J \hat{S}_{1} \hat{S}_{2},
$$

where $J$ is the Heisenberg coupling constant, positive for a ferromagnetic interaction, and $\hat{S}_{1}$ and $\hat{S}_{2}$ are the total spin operators for centers 1 and 2 . This is a purely phenomenological Hamiltonian. Many authors attempted to derive (1) from the exact many-electron nonrelativistic Hamiltonian but a general proof is still lacking except in the asymptotic limit. ${ }^{3}$

The Heisenberg-model Hamiltonian was first introduced to rationalize ferromagnetic interactions. For two electrons, or for two particles with spin $S=\frac{1}{2}$, in two orbitals centered at well-separated nuclei and described by a single spin adapted configuration, one may have a singlet and a triplet electronic states. The triplet energy is the lowest one; the singlet-triplet gap provides the magnitude of the magnetic interaction and is given by the so-called exchange integral. This mechanism is known as direct exchange and $J$ is generally denoted as an exchange constant. However, this simple model cannot account for antiferromagnetic interactions in magnetic-center-ligand-magnetic-center, $M-L-M$, systems where the energy of the singlet is lower. The extension of the Heisenberg model to antiferromagnetic coupling is the Anderson superexchange mechanism. ${ }^{4}$ The basic idea is that one must abandon the single configuration description and go beyond the mean-field approximation. In our example, the superexchange mechanism considers that in addition to the situation where there is one electron per magnetic center [all neutral $M-L-M$ valence-band (VB) components of the electronic wave function] one needs to consider all
$M^{+}-L-M^{-}$instantaneous situations. This is easily generalized if one considers that each VB situation is represented by an electronic configuration. The Anderson model involves the minimum number of configurations that lead to a qualitative description of antiferromagnetism.

The Heisenberg Hamiltonian is commonly used to interpret magnetism in molecular magnets ${ }^{5}$ or extended solids with localized spins. ${ }^{6,7}$ For a molecular magnet the only hypothesis is the reduction of a many-electron system to a net resulting spin. However for an extended system one needs to consider at least the interactions among nearest neighbors and Eq. (1) becomes

$$
\hat{H}^{\text {Heisenberg }}=-\sum_{\langle i, j\rangle} J \hat{S}_{i} \hat{S}_{j},
$$

where the $\langle i, j\rangle$ symbol means summation over nearestneighbor spins (or magnetic centers). Theoretical values for $J$ are obtained by appropriate mapping of the electronic states to the Heisenberg Hamiltonian pure spin eigenfunctions. ${ }^{8,9}$ Similarly, the experimental $J$ values are obtained by fitting experimental data obtained from neutron diffraction, Raman scattering, or magnetic susceptibility using equations derived assuming a suitable Heisenberg model, and spin-orbit and other relativistic effects or small anisotropies can be introduced as corrections to the model. Therefore, the Heisenberg Hamiltonian is the bridge between experiment and theory.

A fundamental question is whether $J$ measures a genuine local two-body interaction or, on the contrary, it is better described as an effective averaged interaction containing the collective effects of the extended system. In the latter case it will be impossible to reproduce the experimental value through a theoretical treatment unless a suitable representation of the extended system is used. The answer to this question is primordial since it has profound implications in the description of the electronic structure of the high- $T_{c}$ superconductors. These materials may exhibit strong antiferromagnetic character ${ }^{10,11}$ and superconductor parent com- 
pounds are also strong antiferromagnets. The electronic structure of these fascinating compounds is that of strongly correlated systems thus precluding the use of the band theory of solids. The theoretical models used to investigate high- $T_{c}$ superconductors often rely on model Hamiltonians which contain parameters such as the Hubbard on-center twoelectron repulsion integral, $U$, the hopping integral, $t$, or the exchange constant $J$. In these model Hamiltonians, the parameters are often extracted from experimental data adding a certain dose of empiricism to these theoretical approaches. ${ }^{12}$ These model theories will strengthen their fundamental basis if the parameters entering into the model Hamiltonian can be deduced from purely theoretical considerations or $a b$ initio calculations.

In this paper we will show that it is possible to use sophisticated quantum chemical calculations to quantitatively reproduce the $J$ experimental values in spite of being often a very small quantity. Moreover, we will show that this is achieved by employing material models containing two magnetic centers only thus opening the way to the $a b$ initio determination of model Hamiltonians. To investigate the local character of the magnetic coupling in strongly correlated systems we have chosen a broad family of compounds. These are $\mathrm{KNiF}_{3}, \mathrm{~K}_{2} \mathrm{NiF}_{4}, \mathrm{KCuF}_{3}, \mathrm{~K}_{2} \mathrm{CuF}_{4}$, and the high- $T_{c}$ parent compound $\mathrm{La}_{2} \mathrm{CuO}_{4}$. This list has been chosen because it includes one-, two-, and three-dimensional (3D) magnetic solids where the magnetic dimensionality corresponds either to the ideal structure or arises from geometric distortion due to the electronic structure, covers a broad class of magnetic coupling going from slightly ferromagnetic to moderately antiferromagnetic and to strongly antiferromagnetic, and includes systems with either $S=1$ or $S=\frac{1}{2}$. For each compound the magnetic coupling constant has been theoretically evaluated by using a cluster model containing a $M_{2} L_{11}$ unit, $M=$ metal $(\mathrm{Cu}$ or $\mathrm{Ni})$ and $L=$ ligand $(\mathrm{F}$ or $\mathrm{O})$, embedded in an adequate environment of total ion potentials which represent the cations near the $M_{2} L_{11}$ unit and an array of point charges which provides a representation of the Madelung potential of the infinite crystal in the cluster region (Fig. 1).

The magnetic coupling constant has been calculated by making use of the univocal relationship existing between spin eigenfunctions of the Heisenberg Hamiltonian and the electronic states of the material model. This one to one correspondence permits us to construct a model space $S$ defined by the $N$-electron basis, or Slater determinants, which, for the material model, expand the spin eigenfunctions that are isomorphic to the spin eigenfunctions of the Heisenberg Hamiltonian. Next, an ab initio effective Hamiltonian $\hat{H}^{\text {eff }}$, acting on the model space $S$, is constructed. Using quasidegenerate-perturbation theory one can show that, up to second order, the $\hat{H}^{\text {eff }}$ matrix elements are given by

$$
\hat{H}_{I, J}^{\text {eff }}=\sum_{K \notin S} \frac{\langle I|\hat{H}| K\rangle\langle K|\hat{H}| J\rangle}{E_{K}^{0}-E_{I}^{0}} ; \quad I, J \in S .
$$

Notice that among the single and double excitations on determinants belonging to $S$, only those $|K\rangle$ determinants simultaneously interacting with $|I\rangle$ and $|J\rangle$ contribute to the off-diagonal elements of $\hat{H}^{\text {eff }}$ and to the energy difference between different electronic states. The $\{|K\rangle\}$ defines the DDCI2 differential space; DDCI stands for difference dedi-

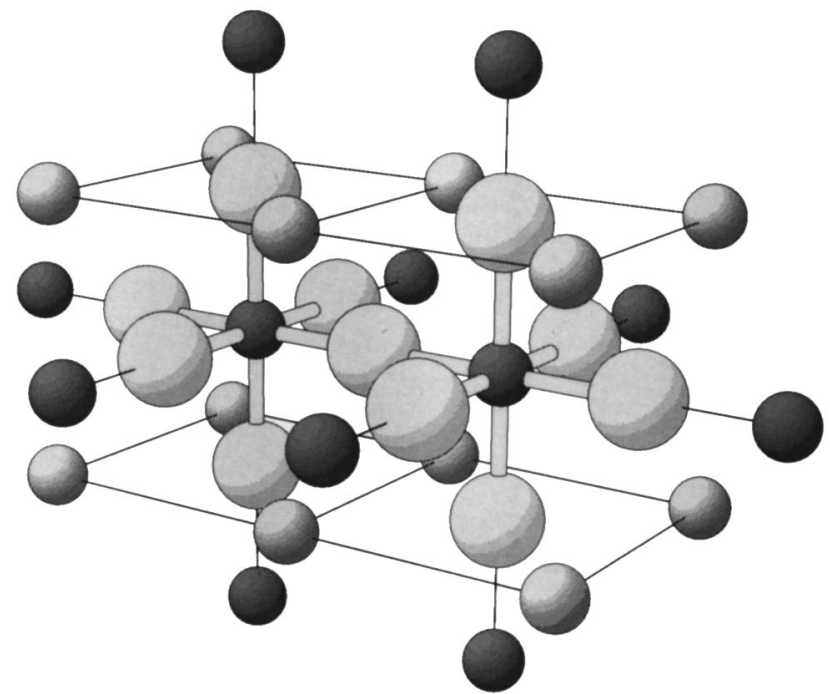

FIG. 1. The $\mathrm{Ni}_{2} \mathrm{~F}_{11}$ cluster model used to represent $\mathrm{KNiF}_{3}$. Similar models are used for the remaining compounds, the only difference being the particular crystal structure. Also shown are the total ion potentials for the nearest $\mathrm{K}^{+}$and $\mathrm{Ni}^{2+}$ cations surrounding the $\mathrm{Ni}_{2} \mathrm{~F}_{11}$ cluster. Thick lines link cluster atoms while thin lines link cluster atom to TIP's; small dark spheres represent $\mathrm{Ni}^{2+}$, small light spheres $\mathrm{K}^{+}$cations, and large spheres $\mathrm{F}^{-}$anions. The overall model is embedded in an array of point charges (not shown) to provide a representation of the Madelung potential.

cated configuration interaction and the 2 indicates that the list only includes excitations, either holes and/or particles, with at most two inactive orbitals (doubly occupied or unoccupied $\forall|I\rangle \in S$ ). The DDCI2 list is in principle sufficient for a correct treatment of magnetic couplings. ${ }^{13}$ When covalent effects are important, the $M-L^{+}-M^{-}$configurations (where $L$ is the ligand bridging atom between the metal atoms) may play an important role in the superexchange mechanism. These charge-transfer configurations are already included in the DDCI2 space as one-hole configurations, but their effective energy is too high unless instantaneous repolarization of these instantaneous physical situations is explicitly accounted for. This effect may be introduced by adding the single excitations of the charge-transfer determinants to the DDCI2 list. The corresponding determinants imply two inactive holes and one inactive particle and belong to an enlarged CI space. This is usually denoted as DDCI3. This enlarged space includes configurations having up to two holes-one particle and one hole-two particles in the inactive orbitals. The present $a b$ initio CI calculations involve the diagonalization of the Hamiltonian matrix representation in the DDCI3 space.

From a technical point of view the Slater determinants have been constructed from a set of one-electron functions, or spin orbitals, which are determined from a suitable $a b$ initio variational mean-field calculation to avoid prejudices in the determination of the magnetic orbitals. This is possible because the spin orbitals are in turn expanded in basis of atomic orbitals expressed as a large linear combination of Gaussian-type functions. ${ }^{14}$ The only external input in the calculation is the geometry of the material model, taken from experimental data, and the set of basis functions and effective core potentials which are derived from atomic $a b$ initio calculations. The expectation value of the energy for the 
TABLE I. Comparison between calculated and experimental values of the magnetic coupling constant for several structures. Acronyms for experimental techniques are as follows: $\mathrm{MS}=$ magnetic susceptibility, ND $=$ neutron diffraction, $\mathrm{RS}=$ Raman scattering; usual experimental error bars are of $\sim 5-10 \%$.

\begin{tabular}{|c|c|c|c|c|}
\hline Compound & $\begin{array}{l}\text { Magnetic } \\
\text { order }\end{array}$ & $\begin{array}{l}\text { Experimental } \\
\text { technique }\end{array}$ & $\begin{array}{l}J / K \text { (exptl.) } \\
\text { value [Ref.] }\end{array}$ & $\begin{array}{c}J / K \text { (calc.) } \\
\text { Present work }\end{array}$ \\
\hline $\mathrm{KNiF}_{3}$ & 3D Heisenberg AFM & MS & $-89[23]$ & -86 \\
\hline \multirow[t]{2}{*}{$\mathrm{K}_{2} \mathrm{NiF}_{4}$} & \multirow[t]{2}{*}{ 2D Heisenberg AFM } & MS & $-95[24]$ & \multirow[t]{2}{*}{-94} \\
\hline & & ND & $-110[24]$ & \\
\hline \multirow[t]{3}{*}{$\mathrm{KCuF}_{3}$} & \multirow[t]{3}{*}{ 1D Heisenberg AFM } & MS & $-380[25]$ & \multirow[t]{3}{*}{-363} \\
\hline & & ND & $-390[26]$ & \\
\hline & & ND & $-406[27]$ & \\
\hline \multirow[t]{2}{*}{$\mathrm{K}_{2} \mathrm{CuF}_{4}$} & \multirow[t]{2}{*}{ 2D Heisenberg FM } & MS & $+17[28]$ & \multirow[t]{2}{*}{+14} \\
\hline & & ND & $+22[29]$ & \\
\hline \multirow[t]{2}{*}{$\mathrm{La}_{2} \mathrm{CuO}_{4}$} & \multirow[t]{2}{*}{ 2D Heisenberg AFM } & $\mathrm{RS}$ & $-1485[30,31]$ & \multirow[t]{2}{*}{-1680} \\
\hline & & ND & $-1555[32]$ & \\
\hline
\end{tabular}

electronic states involved in the evaluation of $J$ is calculated by using the exact, nonrelativistic, Hamiltonian and the DDCI3 wave functions above described. All integrals are explicitly evaluated and a new computer code ${ }^{15}$ has been specifically written to handle the very large expansion defining the wave function (for $\mathrm{Ni}_{2} \mathrm{~F}_{11}$ up to five million determinants are variationally included).

For the present magnetic materials the mechanisms in the Anderson model, energies from a CI calculation involving all the $M-L-M$ and $M^{+}-L-M^{-}$determinants, give rise to qualitative agreement with experiment only. ${ }^{16-18}$ This is because many additional physical mechanisms such as kinetic exchange, double spin polarization, ligand-to-metal and metalto-ligand charge transfer, are neglected. ${ }^{16}$ Explicit consideration of all these effects, which are precisely those defining the DDCI2 list in Eq. (3), up to second order is normally insufficient. Semiquantitative agreement with experiment is achieved when these instantaneous situations are summed up to infinite order, by diagonalizing the Hamiltonian matrix representation in the DDCI2 space. Previous works have shown that these computational schemes permit recovery of roughly $80 \%$ of the magnetic coupling constant in these compounds. ${ }^{8,9,19}$ The remaining difference to experiment can be due to effects arising from the extended nature of these materials or to the neglect of some physical mechanisms related to the electronic correlation effects not included in the DDCI2 computational model. Recent work in many magnetic-center models does not show any noticeable dependence of the calculated $J$ on the number of explicitly interacting magnetic centers. ${ }^{20}$

The clue to the missing effects can be found in the nonorthogonal configuration interaction (NOCI) calculations of Van Oosten et al. ${ }^{21}$ for a series of cuprates. These authors were able to quantitatively reproduce the magnetic coupling constant using two magnetic centers only. This is in agreement with the absence of cluster size effects reported in Ref. 20. The only physical effects that are included in the NOCI approach and not in the previous DDCI2 calculations arise from the instantaneous orbital relaxation for the physical situations where a charge transfer to a magnetic center occurs. Unfortunately, the NOCI approach is computationally too demanding so as to generalize the conclusions to magnetic center with $S \neq \frac{1}{2}$. However, this is readily done in the
DDCI3 approach where all instantaneous situations contributing to these instantaneous orbital relaxation effects are included. This inclusion enormously increases the dimension of the CI matrix with respect to DDCI2 but the results are really worth the effort. In fact, the DDCI3-calculated $J$ values for our family of magnetic compounds completely match the experimental results (Table I). The present theoretical calculations correctly predict the particular orbital ordering ${ }^{22}$ and ferromagnetic behavior of $\mathrm{K}_{2} \mathrm{CuF}_{4}$. An even more quantitative description is achieved either for the weak antiferromagnets, with $J \approx 100 \mathrm{~K}$, or for the strong antiferromagnets with $J$ being an order of magnitude larger. The excellent agreement between purely theoretical and experimental values holds for the 1D, 2D, and 3D magnetic systems. The conclusion from these results is somewhat surprising because it means that in the magnetically ordered wide band-gap insulators, the intersite magnetic coupling arises from essentially local electronic interactions. More precisely it means that in spite of the extended nature of these solids, the magnitude of the magnetic coupling constant does only simultaneously involve the two interacting magnetic centers.

The present paper shows that it is possible to use accurate quantum mechanical calculations to quantitatively predict a rather elusive quantity such as the magnetic coupling constant. Moreover, it shows that this is a local quantity and, hence, opens the way to the accurate ab initio determination of model Hamiltonian parameters which can be used to explain and explore the electronic structure of strongly correlated systems. In conclusion, the present paper suggests that many parameters entering into the definition of currently used model Hamiltonians are in fact purely local and can be obtained from first principles, thus providing strong theoretical support to these theories widely used to investigate the electronic structure of the high- $T_{c}$ superconductors.

Financial support was provided by the Spanish "Ministerio de Educación y Cultura," projects CICyT PB95-0847CO2-01 and PB95-1247. The authors thank CESCA for financial support. I. de P.R.M. is grateful to the University of Barcelona, and C.J.C. to "Ministerio de Educación y Cultura," for financial support. Authors wish to thank T.A. Kaplan for reading the manuscript and pointing out the exact validity of the Heisenberg Hamiltonian in the asymptotic limit. 
${ }^{1}$ W. Heisenberg, Z. Phys. 49, 619 (1928).

${ }^{2}$ P. A. M. Dirac, The Principles of Quantum Mechanics, 3rd ed. (Clarendon, Oxford, 1947), Chap. IX.

${ }^{3}$ C. Herring, Magnetism, edited by G. T. Rado and H. Suhl (Academic, New York, 1965), Vol. 2B.

${ }^{4}$ P. W. Anderson, Phys. Rev. 115, 5745 (1959); Solid State Phys. 14, 99 (1963).

${ }^{5}$ O. Kahn, Molecular Magnetism (VCH, New York, 1993).

${ }^{6}$ N. W. Ashcroft and N. D. Mermin, Solid State Physics (Saunders, Philadelphia, 1976).

${ }^{7}$ C. Kittel, Quantum Theory of Solids (Wiley, New York, 1987).

${ }^{8}$ F. Illas, J. Casanovas, M. A. Garcia-Bach, R. Caballol, and O. Castell, Phys. Rev. Lett. 71, 3549 (1993).

${ }^{9}$ I. de P. R. Moreira and F. Illas, Phys. Rev. B 55, 4129 (1997).

${ }^{10}$ E. Dagotto, in Recent Progress in Many Body Theories, edited by E. Schachinger et al. (Plenum, New York, 1995), Vol. 4.

${ }^{11}$ W. E. Pickett, Rev. Mod. Phys. 61, 433 (1989).

${ }^{12}$ J. Zaanen, G. A. Sawatzky, and J. N. Allen, Phys. Rev. Lett. 55, 418 (1985).

${ }^{13}$ J. Miralles, O. Castell, R. Caballol, and J. P. Malrieu, Chem. Phys. 172, 33 (1993).

${ }^{14}$ For the metal atoms we explicitly include the $3 d, 3 p$, and $3 d$ electrons. The effect of the core electrons is included through a relativistic effective core potential. The metal basis set contains $4 s, 3 p$, and $3 d$ functions. For the ligand bridging the two metal atoms we include all the electrons and use $4 s, 3 p$, and $1 d$ functions. For the rest of the ligands we include the $2 s$ and $2 p$ electrons and use $2 s$ and $2 p$ basis functions. Further details are available upon request to authors.
${ }^{15}$ D. Maynau and N. Ben Amor, CASDI suite of programs.

${ }^{16} \mathrm{Ph}$. de Loth, P. Cassoux, J. P. Daudey, and J. P. Malrieu, J. Am. Chem. Soc. 103, 4007 (1981).

${ }^{17}$ Y. Guo, J. M. Langlois, and W. A. Goddard, III, Science 239, 896 (1988).

${ }^{18}$ G. Chen and W. A. Goddard, III, Science 239, 899 (1988).

${ }^{19}$ J. Casanovas, J. Rubio, and F. Illas, Phys. Rev. B 53, 945 (1996).

${ }^{20}$ F. Illas, I. de P. R. Moreira, C. de Graaf, O. Castell, and J. Casanovas, Phys. Rev. B 56, 5069 (1997).

${ }^{21}$ A. B. Van Oosten, R. Broer, and W. C. Nieuwpoort, Chem. Phys. Lett. 257, 207 (1996).

${ }^{22}$ Magnetic coupling in $\mathrm{KCuF}_{3}$ and $\mathrm{K}_{2} \mathrm{CuF}_{4}$ involves a particular orbital ordering that was earlier suggested by D. Khomskii and K. I. Kugel' in Solid State Commun. 13, 763 (1973). The magnetic orbitals used in the present calculations exhibit this orbital ordering. An exhaustive study of these two compounds will be reported in the near future.

${ }^{23}$ M. E. Lines, Phys. Rev. 164, 736 (1967).

${ }^{24}$ L. J. de Jongh and R. Miedema, Adv. Phys. 23, 1 (1974).

${ }^{25}$ S. Kadota et al., J. Phys. Soc. Jpn. 23, 751 (1967).

${ }^{26}$ M. T. Hutchings, E. J. Samuelsen, G. Shirane, and K. Hirakawa, Phys. Rev. 188, 919 (1969).

${ }^{27}$ S. K. Satija et al., Phys. Rev. B 21, 2001 (1980).

${ }^{28}$ I. Yamada, J. Phys. Soc. Jpn. 33, 979 (1972).

${ }^{29}$ K. Hirakawa et al., J. Phys. Soc. Jpn. 35, 1328 (1973).

${ }^{30}$ R. R. P. Singh et al., Phys. Rev. Lett. 62, 2736 (1989).

${ }^{31}$ P. E. Sulewski, P. A. Fleury, K. B. Lions, S.-W. Cheong, and Z. Fisk, Phys. Rev. B 41, 225 (1990).

${ }^{32}$ G. Aeppli et al., Phys. Rev. Lett. 62, 2052 (1989). 